\title{
TMDs and Drell-Yan Experiments at Fermilab and J-PARC
}

\author{
Jen-Chieh Peng \\ Department of Physics, University of Illinois at Urbana-Champaign \\ Urbana, IL 61801, U.S.A. \\ E-mail: jcpeng@illinois.edu
}

\begin{abstract}
The roles of the Drell-Yan experiments in studying the Transverse-MomentumDependent (TMD) parton distributions are discussed. Recent results from the Fermilab E866 experiment on the angular distributions of Drell-Yan dimuons in $p+p$ and $p+d$ at $800 \mathrm{GeV} / \mathrm{c}$ are presented. These data are compared with the pion-induced Drell-Yan data, and with models which attribute the $\cos 2 \phi$ azimuthal distribution to the presence of the transverse-momentum-dependent Boer-Mulders structure function $h_{1}^{\perp}$. Constraints on the magnitude of the seaquark $h_{1}^{\perp}$ structure functions are obtained. Future prospects for studying the TMDs with Drell-Yan experiments at Fermilab and J-PARC are also discussed.
\end{abstract}

Keywords: Drell-Yan, azimuthal asymmetry, Boer-Mulders functions

\section{Introduction}

While our current knowledge on the partonic substructure of nucleons is obtained largely from deep-inelastic scattering (DIS) experiments, the DrellYan process, ${ }^{1}$ in which a charged lepton pair is produced in a hadron-hadron interaction via the electromagnetic $q \bar{q} \rightarrow l^{+} l^{-}$process, also provides unique information on parton distributions. In particular, the Drell-Yan process has been used to determine the antiquark contents of nucleons and nuclei, ${ }^{2}$ as well as the quark distributions of pions, kaons, and antiprotons. ${ }^{3}$ Such information is difficult, if not impossible, to obtain from DIS experiments. As the Drell-Yan process can be well described by next-to-leading order QCD calculations, ${ }^{4}$ a firm theoretical framework exists for utilizing the Drell-Yan process to extract the parton distributions.

The study of the transverse momentum dependent (TMD) parton distributions of the nucleon has received much attention in recent years as it provides new perspectives on the hadron structure and QCD. ${ }^{5}$ These novel TMDs can be extracted from semi-inclusive deep-inelastic (SIDIS) scattering experiments. Recent measurements of the SIDIS by the HERMES ${ }^{6}$ and 
COMPASS $^{7}$ collaborations have shown clear evidence for the existence of the T-odd Sivers functions. These data also allow the first determination ${ }^{8}$ of the magnitude and flavor structure of the Sivers functions and the nucleon transversity distributions.

The TMD and transversity parton distributions can also be probed in Drell-Yan experiments. As pointed out ${ }^{9}$ long time ago, the double transverse spin asymmetry in polarized Drell-Yan, $A_{T T}$, is proportional to the product of transversity distributions, $h_{1}\left(x_{q}\right) h_{1}\left(x_{\bar{q}}\right)$. The single transverse spin asymmetry, $A_{N}$, is sensitive to the Sivers function, ${ }^{10} f_{1 T}^{\perp}(x)$ of the polarized proton (beam or target). Even unpolarized Drell-Yan experiments can be used to probe the TMD distribution function, since the $\cos 2 \phi$ azimuthal angular dependence is proportional to the product of two BoerMulders functions, ${ }^{11} h_{1}^{\perp}\left(x_{1}\right) \bar{h}_{1}^{\perp}\left(x_{2}\right)$. A unique feature of the Drell-Yan process is that, unlike the SIDIS, no fragmentation functions are involved. Therefore, the Drell-Yan process provides an entirely independent technique for measuring the TMD functions. Furthermore, the proton-induced Drell-Yan process is sensitive to the sea-quark TMDs and can lead to flavor separation of TMDs when combined with the SIDIS data. Finally, the intriguing prediction ${ }^{12}$ that the T-odd TMDs extracted from DIS will have a sign-change for the Drell-Yan process remains to be tested experimentally.

In this article, we first present the measurement of Drell-Yan azimuthal angular distributions in the Fermilab experiment E866. Implication of the data on the TMD Boer-Mulders function is discussed. Prospects for future Drell-Yan experiments for studying TMD and transversity distributions at Fermilab and J-PARC will also be discussed.

\section{Fermilab dimuon experiments and TMDs}

\subsection{Overview}

During the last two decades, a series of fixed-target dimuon production experiments (E772, E789, E866) have been carried out using $800 \mathrm{GeV} / \mathrm{c}$ proton beam at Fermilab. At $800 \mathrm{GeV} / \mathrm{c}$, the dimuon data contain Drell-Yan continuum up to dimuon mass of $\sim 15 \mathrm{GeV}$ as well as quarkonium $(\mathrm{J} / \Psi$, $\Psi^{\prime}$, and $\Upsilon$ resonances) productions. The Drell-Yan process and quarkonium productions often provide complementary information, since Drell-Yan is an electromagnetic process via quark-antiquark annihilation while the quarkonium production is a strong interaction process dominated by gluon-gluon fusion at this beam energy.

The Fermilab dimuon experiments cover a broad range of physics top- 
ics. The Drell-Yan data have provided informations on the antiquark distributions in the nucleons ${ }^{13-16}$ and nuclei. ${ }^{17,18}$ These results showed the surprising results that the antiquark distributions in the nuclei are not enhanced, ${ }^{17,18}$ contrary to the predictions of models which explain the EMC effect in term of nuclear enhancement of exchanged mesons. Moreover, the Drell-Yan cross section ratios $(p+d) /(p+p)$ clearly establish the flavor asymmetry of the $\bar{d}$ and $\bar{u}$ distributions in the proton, and they map out the $x$-dependence of this asymmetry. ${ }^{14-16} \mathrm{~A}$ recent analysis of the $\Upsilon$ production cross section ratios for $(p+d) /(p+p)$ has shown that the gluon distributions in proton and neutron are very similar. ${ }^{19}$ Pronounced nuclear dependences of quarkonium productions as a function of $x_{F}$ and $p_{T}$ have been observed for $\mathrm{J} / \Psi, \Psi^{\prime}$, and $\Upsilon$ resonances.$^{20-23}$ The nuclear Drell-Yan cross sections also exhibit $x_{F}$ as well as $p_{T}$ dependences, ${ }^{2,18,24,25}$ which are weaker than the quarkonium nuclear dependences but can provide information on the energy loss of quarks traversing the nucleus. ${ }^{26}$

Several review articles covering some of these results are available. ${ }^{2,27,28}$ In the following, we will focus on the recent results from experiment E866 on the measurement of angular distributions of the Drell-Yan cross sections.

\subsection{Angular distributions of Drell-Yan and the Boer-Mulders function}

Despite the success of perturbative QCD in describing the Drell-Yan cross sections, it remains a challenge to understand the angular distributions of the Drell-Yan process. Assuming dominance of the single-photon process, a general expression for the Drell-Yan angular distribution is ${ }^{29}$

$$
\frac{d \sigma}{d \Omega} \propto 1+\lambda \cos ^{2} \theta+\mu \sin 2 \theta \cos \phi+\frac{\nu}{2} \sin ^{2} \theta \cos 2 \phi,
$$

where $\theta$ and $\phi$ denote the polar and azimuthal angle, respectively, of the $\mathrm{l}^{+}$in the dilepton rest frame. In the "naive" Drell-Yan model, where the transverse momentum of the quark is ignored and no gluon emission is considered, $\lambda=1$ and $\mu=\nu=0$ are obtained. QCD effects ${ }^{30}$ and non-zero intrinsic transverse momentum of the quarks ${ }^{31}$ can both lead to $\lambda \neq 1$ and $\mu, \nu \neq 0$. However, $\lambda$ and $\nu$ should still satisfy the relation $1-\lambda=2 \nu{ }^{29}$ This so-called Lam-Tung relation, obtained as a consequence of the spin$1 / 2$ nature of the quarks, is analogous to the Callan-Gross relation ${ }^{32}$ in deep-inelastic scattering.

The first measurement of the Drell-Yan angular distribution was performed by the NA10 Collaboration for $\pi^{-}+W$ with the highest statistics 
at $194 \mathrm{GeV} / \mathrm{c}^{33,34}$ The $\cos 2 \phi$ angular dependences showed a sizable $\nu$, increasing with dimuon transverse momentum $\left(p_{T}\right)$ and reaching a value of $\approx 0.3$ at $p_{T}=2.5 \mathrm{GeV} / \mathrm{c}$. The Fermilab E615 Collaboration subsequently performed a measurement of $\pi^{-}+W$ Drell-Yan production at $252 \mathrm{GeV} / \mathrm{c}$ with broad coverage in the decay angle $\theta{ }^{35}$ The E615 data showed that the Lam-Tung relation, $2 \nu=1-\lambda$, is clearly violated.

The NA10 and E615 results on the Drell-Yan angular distributions strongly suggest that new effects beyond conventional perturbative QCD are present. Brandenburg, Nachtmann and Mirke suggested that a factorization-breaking QCD vacuum may lead to a correlation between the transverse spin of the antiquark in the pion and that of the quark in the nucleon. ${ }^{36}$ This would result in a non-zero $\cos 2 \phi$ angular dependence consistent with the data. Several authors have also considered higher-twist effects from quark-antiquark binding in pions. ${ }^{37,38}$ However, the model is strictly applicable only in the $x_{\pi} \rightarrow 1$ region, while the NA10 and E615 data exhibit nonperturbative effects over a much broader kinematic region.

More recently, Boer pointed out ${ }^{39}$ that the $\cos 2 \phi$ angular dependences observed in NA10 and E615 could be due to the $k_{T}$-dependent parton distribution function $h_{1}^{\perp}$. This so-called Boer-Mulders function ${ }^{11}$ is an example of a novel type of $k_{T}$-dependent parton distribution function, and it characterizes the correlation of a quark's transverse spin and its transverse momentum, $k_{T}$, in an unpolarized nucleon. The Boer-Mulders function is the chiral-odd analog of the Sivers function and owes its existence to the presence of initial/final state interactions. ${ }^{40}$ While the Sivers function is beginning to be quantitatively determined from the SIDIS experiments, very little is known about the Boer-Mulders function so far.

Several model calculations have been carried out for the Boer-Mulders functions. In the quark-diquark model, it was shown that the Boer-Mulders functions are identical to the Sivers functions when only the scalar diquark configuration is considered. ${ }^{40,41}$ More recently, calculations taking into account both the scalar and the axial-vector diquark configurations found significant differences between the Sivers and Boer-Mulders functions. ${ }^{42}$ In particular, the $u$ and $d$ quark Boer-Mulders functions are predicted to be both negative, while the Sivers function is negative for the $u$ quark and positive for the $d$ quark. Other calculations using the MIT bag model, ${ }^{43}$ the relativistic constituent quark model, ${ }^{44}$ the large- $N_{c}$ model,,${ }^{45}$ and the lattice $\mathrm{QCD}^{46}$ also predicted negative signs for the $u$ and $d$ Boer-Mulders functions. Burkardt recently pointed out ${ }^{47}$ that the origin of the negative signs for the Boer-Mulders functions lies in the phase of the $p$-wave lower 
component relative to the $s$-wave upper component of the quark wave function for the solution to the free Dirac equation. The model predictions for the same signs of the $u$ and $d$ Boer-Mulders functions remain to be tested experimentally.

\subsection{Results from Fermilab E866}

To shed additional light on the origins of the NA10 and E615 Drell-Yan angular distributions, we recently analyzed $p+p$ and $p+d$ Drell-Yan angular distribution data at $800 \mathrm{GeV} / \mathrm{c}$ from Fermilab E866. There had been no report on the azimuthal angular distributions for proton-induced DrellYan - all measurements were for polar angular distributions. ${ }^{2,48,49}$ Protoninduced Drell-Yan data provide a test of theoretical models. For example, the $\cos 2 \phi$ dependence is expected to be much reduced in proton-induced Drell-Yan if the underlying mechanism involves the Boer-Mulders functions. This is due to the expectation that the Boer-Mulders functions are small for the sea-quarks. ${ }^{50}$ However, if the QCD vacuum effect ${ }^{36}$ is the origin of the $\cos 2 \phi$ angular dependence, then the azimuthal behavior of proton-induced Drell-Yan should be similar to that of pion-induced Drell-Yan. Finally, the validity of the Lam-Tung relation has never been tested for proton-induced Drell-Yan, and the present study provides a first test.

The Fermilab E866 experiment was performed using the upgraded Meson-East magnetic pair spectrometer. An $800 \mathrm{GeV} / \mathrm{c}$ primary proton beam with up to $2 \times 10^{12}$ protons per 20 -second beam spill was incident upon $50.8 \mathrm{~cm}$ long target flask containing either liquid hydrogen, liquid deuterium or vacuum. The detector system consisted of four tracking stations and a momentum analyzing magnet. From the momenta of the $\mu^{+}$ and $\mu^{-}$, kinematic variables of the dimuons $\left(x_{F}, m_{\mu \mu}, p_{T}\right)$ were readily reconstructed. The muon angles $\theta$ and $\phi$ in the Collins-Soper frame ${ }^{51}$ were also calculated. To remove the quarkonium background, only events with $4.5<m_{\mu \mu}<9 \mathrm{GeV} / \mathrm{c}^{2}$ or $m_{\mu \mu}>10.7 \mathrm{GeV} / \mathrm{c}^{2}$ were analyzed. A total of $\sim 54,000 p+p$ and $\sim 118,000 \mathrm{p}+\mathrm{d}$ Drell-Yan events covering the decay angular range $-0.5<\cos \theta<0.5$ and $-\pi<\phi<\pi$ remain.

The measurement of the $\cos 2 \phi$ dependence for the $p+d$ Drell-Yan cross sections has already been reported. ${ }^{52}$ As shown in Fig. 1, significantly smaller (but non-zero) $\cos 2 \phi$ azimuthal angular dependence was observed in the $p+d$ reaction. While pion-induced Drell-Yan process is dominated by annihilation between a valence antiquark in the pion and a valence quark in the nucleon, proton-induced Drell-Yan process involves a valence quark annihilating with a sea antiquark in the nucleon. Therefore, the $p+d$ result 
suggests $^{52}$ that the Boer-Mulders functions for sea antiquarks are significantly smaller than those for valence quarks.

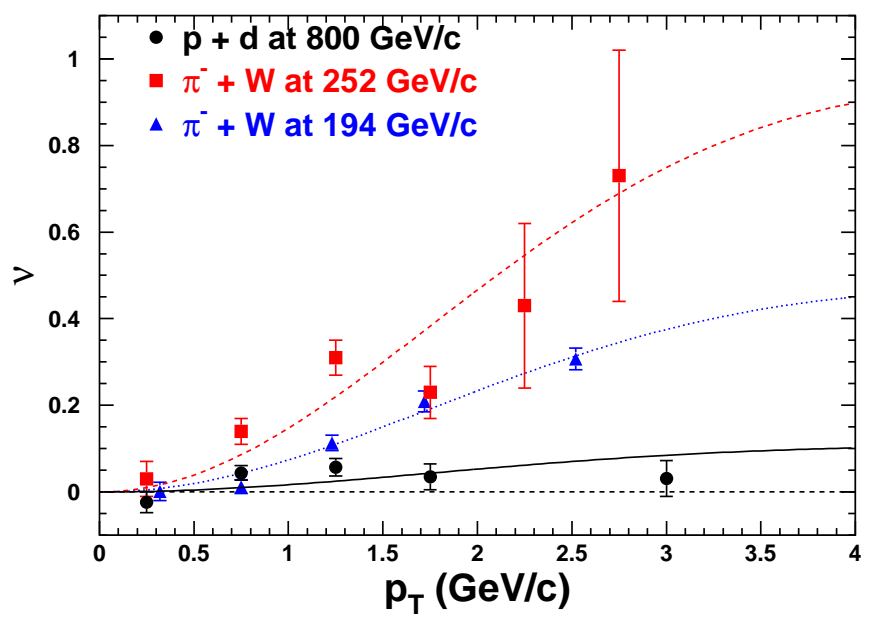

Fig. 1. Parameter $\nu$ vs. $p_{T}$ in the Collins-Soper frame for three Drell-Yan measurements. Fits to the data using an empirical expression ${ }^{39}$ are also shown.

A recent analysis ${ }^{53}$ of the $p+d \cos 2 \phi$ data showed that the sea-quark Boer-Mulders functions are indeed smaller by a factor $\sim 5$ than the valencequark Boer-Mulders functions. This analysis also indicated that the E866 $p+d$ data are consistent with the $u$ and $d$ Boer-Mulders functions having the same signs, as predicted by various models. However, the $p+d$ data alone can not provide an unambiguous determination of the flavor dependence of the Boer-Mulders functions. Moreover, it was recently pointed out ${ }^{54,55}$ that QCD processes would lead to sizeable $\cos 2 \phi$ effect which has not been taken into account in the extractions ${ }^{39,53,56}$ of Boer-Mulders functions from the Drell-Yan data. The $p+p$ Drell-Yan data should provide further constraints on the flavor dependence of the Boer-Mulders functions. ${ }^{56}$ It is also interesting to compare the $p+p$ and $p+d$ data with the prediction of QCD.

Figure 2 shows the angular distribution parameters $\lambda, \mu$, and $\nu$ vs. $p_{T}$ for the $\mathrm{E} 866 p+p$ and $p+d$ Drell-Yan data. Within statistics, the angular distributions of $p+p$ are consistent with those of $p+d$. Also shown in Fig. 2 is the quantity $2 \nu-(1-\lambda)$, which should vanish if the Lam-Tung relation is valid. Figure 2 shows that the Lam-Tung relation is indeed quite well satisfied within statistics. This is different from the observation of a 
significant violation of the Lam-Tung relation by the E615 collabortaion in the $\pi^{-}+W$ reaction at $252 \mathrm{GeV} /$ c. $^{35}$

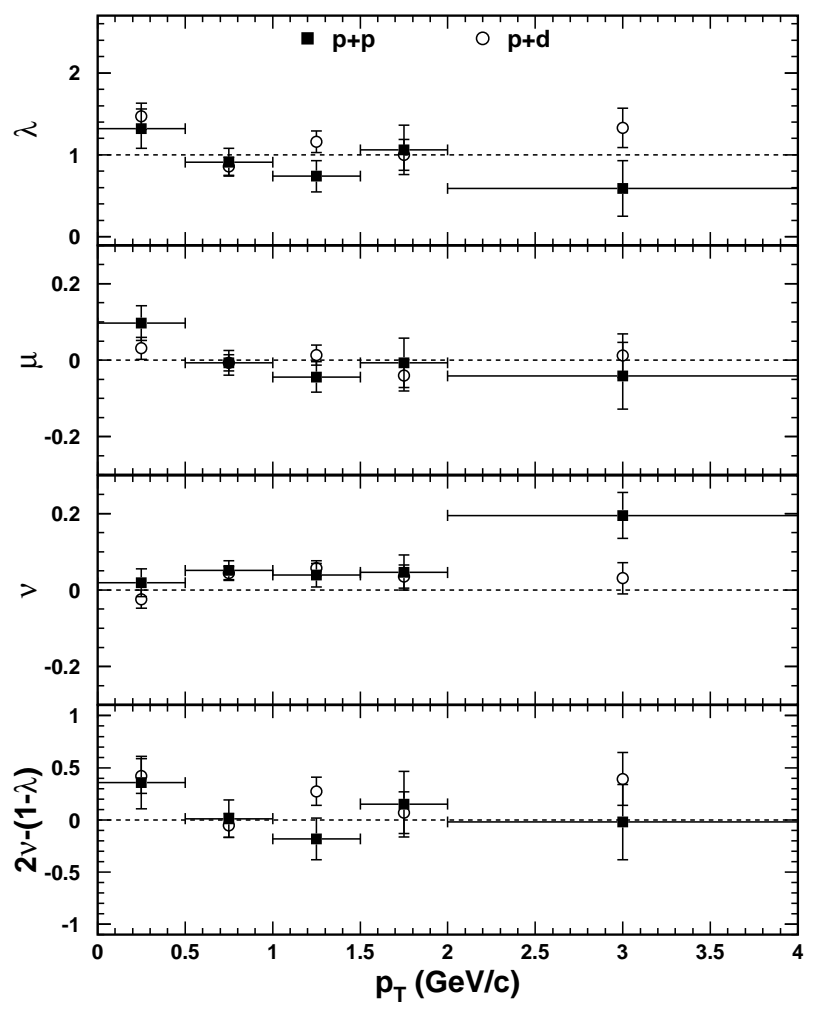

Fig. 2. Parameters $\lambda, \mu, \nu$ and $2 \nu-(1-\lambda)$ vs. $p_{T}$ in the Collins-Soper frame. Solid squares (open circles) are for E866 $p+p(p+d)$ at $800 \mathrm{GeV} / \mathrm{c}$.

Figure 3 shows the parameter $\nu$ vs. $p_{T}$ for the $p+p$ and $p+d$ DrellYan data. The solid curves are calculations ${ }^{53,56}$ for $p+p$ and $p+d$ using parametrizations based on a fit to the $p+d$ Drell-Yan data. The larger values of $\nu$ for $p+p$ compared to $p+d$ are in qualitative agreement with the prediction. However, the shape of the predicted $p_{T}$ dependence is different from the data. This strongly suggests that there could be other mechanisms contributing to the $\cos 2 \phi$ azimuthal angular dependence at large $p_{T}$ region. In a recent paper, ${ }^{55}$ the QCD contribution to the $\cos 2 \phi$ azimuthal angular 
dependence is given as

$$
\nu=\frac{Q_{\perp}^{2} / Q^{2}}{1+\frac{3}{2} Q_{\perp}^{2} / Q^{2}}
$$

where $Q_{\perp}$ is the dimuon transverse momentum. The predicted QCD contribution is shown as the dotted curve is Fig. 3. Although neither the prediction based on Boer-Mulders functions nor the QCD prediction could describe the entire range of $p_{T}$, it is evident that the QCD contribution becomes important at high $p_{T}$ and the Boer-Mulders functions contribute at lower $p_{T}$. An analysis combining both effects is required before a reliable extraction of the Boer-Mulders functions could be obtained.

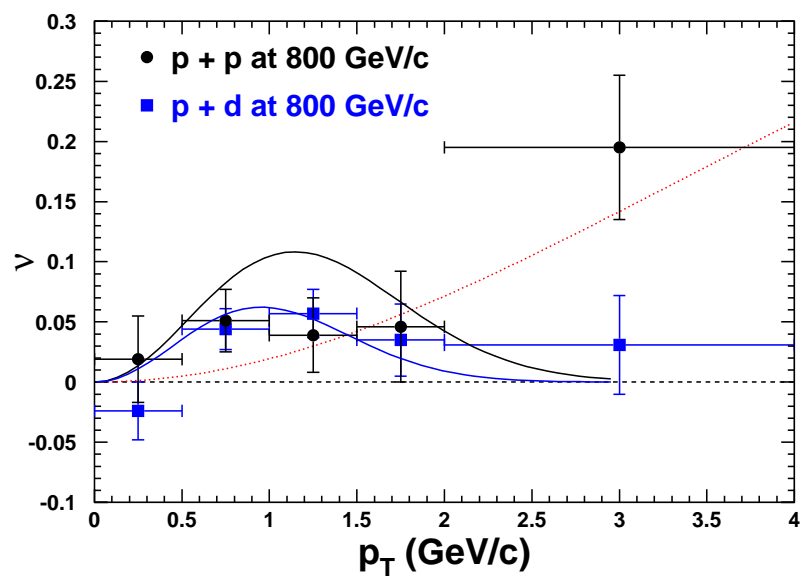

Fig. 3. Parameter $\nu$ vs. $p_{T}$ for the $p+p$ and $p+d$ Drell-Yan data. The solid curves are calculations ${ }^{53}$ for $p+p$ and $p+d$ using parametrizations based on a fit to the $p+d$ Drell-Yan data. The dotted curve is the contribition from QCD process (Eq. 2).

\section{Future Prospects at Fermilab and J-PARC}

Future fixed-target dimuon experiments have been proposed at the 120 GeV Fermilab Main Injector (FMI) and the $50 \mathrm{GeV}$ J-PARC facilities. The Fermilab proposal, ${ }^{57}$ E906, has been approved and is expected to start data-taking around 2011. Two dimuon proposals (P04 ${ }^{58}$ and $\mathrm{P} 24^{59}$ ) have also been submitted to the J-PARC for approval. The lower beam energies 
at FMI and J-PARC present opportunities for extending the Drell-Yan measurements to larger $x(x>0.25)$. For given values of $x_{1}$ and $x_{2}$, the Drell-Yan cross section is proportional to $1 / \mathrm{s}$, hence a gain of $\sim 16$ times in the Drell-Yan cross sections can be obtained at the J-PARC energy of $50 \mathrm{GeV}$. This would extend the measurement of Boer-Mulders functions to larger $x$. In addition, the nuclear dependence of the Boer-Mulders functions can also be measured.

The dimuon physics program at J-PARC is proposed to be carried out in several stages. Since $30 \mathrm{GeV}$ proton beam will be available at the initial phase of J-PARC, the first measurements will focus on $J / \Psi$ production at $30 \mathrm{GeV}$. This will be followed by measurements of Drell-Yan and quarkonium production at $50 \mathrm{GeV}$ after the beam energy is upgraded to $50 \mathrm{GeV}$. Experiments using polarized target could already be performed with unpolarized beams. When polarized proton beam becomes available at J-PARC, a rich and unique program on spin physics could also be pursued at J-PARC using the dimuon spectrometer.

An important feature of $J / \Psi$ production using 30 or $50 \mathrm{GeV}$ proton beam is the dominance of the quark-antiquark annihilation subprocess. This is in striking contrast to $J / \Psi$ production at $800 \mathrm{GeV}$ (Fermilan E866) or at $120 \mathrm{GeV}$ (Fermilab E906), where the gluon-gluon fusion is the dominant process. This suggests an exciting opportunity to use $J / \Psi$ production at J-PARC as an alternative method to probe antiquark distribution.

With the possibility to accelerate polarized proton beams at J-PARC, the spin structure of the proton can also be investigated with the proposed dimuon experiments. In particular, polarzied Drell-Yan process with polarized beam and/or polarized target at J-PARC would allow a unique program on spin physics complementary to polarized DIS experiments and the RHIC-Spin program. Specific physics topics include the measurements of T-odd Boer-Mulders distribution function in unpolarized Drell-Yan, the extraction of T-odd Sivers distribution functions in singly transversely polarized Drell-Yan, the helicity distribution of antiqaurks in doubly longitudinally polarized Drell-Yan, and the transversity distribution in doubly transversely polarized Drell-Yan. It is worth noting that polarized Drell-Yan is one of the major physics program at the GSI Polarized Antiproton Experiment (PAX). The RHIC-Spin program will likely provide the first results on polarized Drell-Yan. However, the high luminosity and the broad kinematic coverage for the large- $x$ region at J-PARC would allow some unique measurements to be performed in the J-PARC dimuon experiments. 


\section{Acknowledgments}

I am very grateful to Lingyan Zhu, Paul Reimer, and my collaborators on the E772, E789, and E866 experiments at Fermilab. Illuminating comments from Matthias Burkardt, Feng Yuan, Bo-Qiang Ma, Werner Vogelsang, and Jianwei Qiu are greatly appreciated. I would also like to acknowledge the collaboration with Shin'ya Sawada and Yuji Goto on the J-PARC dimuon proposals.

\section{References}

1. S.D. Drell and T.M. Yan, Phys. Rev. Lett. 25, 316 (1970); Ann. Phys. (NY) 66, 578 (1971).

2. P.L. McGaughey, J.M. Moss, and J.C. Peng, Annu. Rev. Nucl. Part. Sci. 49, 217 (1999).

3. I.R. Kenyon, Rep. Prog. Phys. 45, 1261 (1982); K. Freudenreich, Int. J. Mod. Phys. A5, 3643 (1990).

4. W.J. Stirling and M.R. Whalley, J. Phys. G19, D1 (1993).

5. V. Barone, A. Drago, and P. G. Ratcliffe, Phys. Rep. 359, 1 (2002).

6. HERMES Collaboration, A. Airapetian et al., Phys. Rev. Lett. 94, 012002 (2005); M. Diefenthaler, arXiv: 0706.2242.

7. COMPASS Collaboration, V. Yu. Alexakhin et al., Phys. Rev. Lett. 94, 202002 (2005); M. Alekseev et al., arXiv: 0802.2160.

8. W. Vogelsang and F. Yuan, Phys. Rev. D 72, 054028 (2005); M. Anselmino et al., Phys. Rev. D 72, 094007 (2005); M. Anselmino et al., arXiv:0807.0166.

9. J.P. Ralston and D.E. Soper, Nucl. Phys. B 152, 109 (1979).

10. D. Sivers, Phys. Rev. D 41, 83 (1990).

11. D. Boer and P.J. Mulders, Phys. Rev. D 57, 5780 (1998).

12. J.C. Collins, Phys. Lett. B 536, 43 (2002).

13. E772 Collaboration, P.L. McGaughey et al., Phys. Rev. Lett. 69, 1726 (1992).

14. E866 Collaboration, E.A. Hawker et al., Phys. Rev. Lett. 80, 3715 (1998).

15. E866 Collaboration, J.C. Peng et al., Phys. Rev. D 58, 092004 (1998).

16. E866 Collaboration, R.S. Towell et al., Phys. Rev. D 64, 052002 (2001).

17. E772 Collaboration, D.M. Alde et al., Phys. Rev. Lett. 64, 2479 (1990).

18. E866 Collaboration, M.A. Vasiliev et al., Phys. Rev. Lett. 83, 2304 (1999).

19. E866 Collaboration, L.Y. Zhu et al., Phys. Rev. Lett. 100, 062301 (2008).

20. E772 Collaboration, D.M. Alde et al., Phys. Rev. Lett. 66, 133 (1991).

21. E772 Collaboration, D.M. Alde et al., Phys. Rev. Lett. 66, 2285 (1991).

22. E789 Collaboration, M.S. Kowitt et al., Phys. Rev. Lett. 72, 1318 (1994).

23. E789 Collaboration, M.J. Leitch et al., Phys. Rev. D 52, 4251 (1995).

24. E772 Collaboration, M.B. Johnson et al., Phys. Rev. Lett. 86, 4483 (2001).

25. M.B. Johnson et al., Phys. Rev. C 65, 025203 (2002).

26. G.T. Garvey and J.C. Peng, Phys. Rev. Lett. 90, 092302 (2003).

27. G.T. Garvey and J.C. Peng, Prog. Part. Nucl. Phys. 47, 203 (2001).

28. P.E. Reimer, J. Phys. G 34, S107 (2007).

29. C.S. Lam and W.K. Tung, Phys. Rev. D 18, 2447 (1978). 
30. P. Chiappetta and M. LeBellac, Z. Phys. C 32, 521 (1986).

31. J. Cleymans and M. Kuroda, Phys. Lett. B 105, 68 (1981).

32. C.G. Callan and D. J. Gross, Phys. Rev. Lett. 22, 156 (1969).

33. NA10 Collaboration, S. Falciano et al., Z. Phys. C 31, 513 (1986).

34. NA10 Collaboration, M. Guanziroli et al., Z. Phys. C 37, 545 (1988).

35. E615 Collaboration, J.S. Conway et al., Phys. Rev. D 39, 92 (1989); J.G. Heinrich et al., Phys. Rev. D 44, 1909 (1991).

36. A. Brandenburg, O. Nachtmann, and E. Mirkes, Z. Phys. C 60, 697 (1993).

37. A. Brandenburg, S.J. Brodsky, V.V. Khoze, and D. Müller, Phys. Rev. Lett. 73, 939 (1994)

38. K.J. Eskola, P. Hoyer, M. Väntinnen, and R. Vogt, Phys. Lett. B 333, 526 (1994).

39. D. Boer, Phys. Rev. D 60, 014012 (1999).

40. D. Boer, S.J. Brodsky, and D.S. Hwang, Phys. Rev. D 67, 054003 (2003).

41. L.P. Gamberg, G.R. Goldstein, and K.A. Oganessyan, Phys. Rev. D 67, 071504(R) (2003); A. Bacchetta, A. Schäfer, and J.-J. Yang, Phys. Lett. B 578, 109 (2004).

42. L.P. Gamberg, G.R. Goldstein, and M. Schlegel, Phys. Rev. D 77, 094016 (2008).

43. F. Yuan, Phys. Lett. B 575, 45 (2003).

44. B. Pasquini, M. Pincetti, and S. Boffi, Phys. Rev. D 76, 034020 (2007).

45. P.V. Pobylitsa, hep-ph/0301236.

46. M. Göckeler et al., Phys. Rev. Lett. 98, 222001 (2007).

47. M. Burkardt and B. Hannafious, Phys. Lett. B 658, 130 (2008).

48. E866 Collaboration, T.H. Chang et al., Phys. Rev. Lett. 91, 211801 (2003).

49. E866 Collaboration, C.N. Brown et al., Phys. Rev. Lett. 86, 2529 (2001).

50. Z. Lu, B.-Q. Ma, and I. Schmidt, Phys. Rev. D 75, 014026 (2007).

51. J.C. Collins and D.E. Soper, Phys. Rev. D16, 2219 (1977).

52. E866 Collaboration, L.Y. Zhu et al., Phys. Rev. Lett. 99, 082301 (2007).

53. B. Zhang, Z. Lu, B.-Q. Ma, and I. Schmidt, Phys. Rev. D 77, 054011 (2008).

54. D. Boer and W. Vogelsang, Phys. Rev. D 74, 014004 (2006).

55. E.L. Berger, J.-W. Qiu, and R.A. Rodriguez-Pedraza, Phys. Lett. B 656, 74 (2007); Phys. Rev. D 76, 074006 (2007).

56. B. Zhang, Z. Lu, B.-Q. Ma, and I. Schmidt, arXiv: 0807.0503.

57. http://www.phy.anl.gov/mep/drell-yan; D. Geesaman, P. Reimer, et al., Fermilab Proposal P906 (1999).

58. http://j-parc.jp/NuclPart/pac_0606/pdf/p04-Peng.pdf; J. Peng, S. Sawada, et al., J-PARC Proposal P04 (2006).

59. http://j-parc.jp/NuclPart/pac_0801/pdf/Goto.pdf; Y. Goto, H. Sato, et al., J-PARC Proposal P24 (2007). 